\title{
La Mairie avant la République : les bâtiments municipaux de l'arrondissement de Fougères au XIXe siècle
}

\section{Denis Geffroy}

\section{(2) OpenEdition \\ 1 Journals}

\section{Édition électronique}

URL : http://journals.openedition.org/abpo/1474

DOI : $10.4000 / a b p o .1474$

ISBN : 978-2-7535-1489-8

ISSN : 2108-6443

\section{Éditeur}

Presses universitaires de Rennes

\section{Édition imprimée}

Date de publication : 20 mars 2003

Pagination : $125-143$

ISBN : 978-2-86847-811-5

ISSN : 0399-0826

\section{Référence électronique}

Denis Geffroy, «La Mairie avant la République : les bâtiments municipaux de l'arrondissement de Fougères au XIXe siècle ", Annales de Bretagne et des Pays de l'Ouest [En ligne], 110-1 | 2003, mis en ligne le 20 mars 2005, consulté le 19 avril 2019. URL : http://journals.openedition.org/abpo/1474 ; DOI : 10.4000/abpo.1474 


\title{
La Mairie avant la République : les bâtiments municipaux de l'arrondisse- ment de Fougères au XIX ${ }^{\mathrm{e}}$ siècle
}

\author{
Denis GeFrROY \\ Étudiant à l'Institut d'Études politiques de Paris
}

«La mairie est républicaine par définition [...] puisque c'est précisément à la République que l'on doit l'universalité de sa présence ${ }^{1}$."

C'est une idée communément admise que d'attribuer à la Troisième République la construction, dans chaque village de France, d'une mairie, généralement associée à l'école communale. Présence universelle puisque la loi municipale du 5 avril 1884 rend obligatoire pour chaque commune " l'entretien de l'hôtel de ville, ou, si la commune n'en possède pas, la location d'une maison ou d'une salle pour en tenir lieu ${ }^{2}$ ». Pourtant, Jocelyne George constate que « la République n'a pas, comme par magie, répandu sur la France maisons communes et salles de classe. Elle a complété, magnifié un équipement entrepris par les maires de la monarchie de Juillet ${ }^{3}$ ". De même Robert Liris estime que " le début du mouvement de constructions civiles et utilitaires peut se placer sous la Monarchie de Juillet ${ }^{4}$ ". Ces appréciations suggèrent donc que le phénomène est plus ancien et plus complexe que ne le laisse à penser l'historiographie. Mais elles partagent avec cette dernière un déficit d'études précises, déficit paradoxal pour un bâtiment si familier. En effet, Maurice Agulhon reconnaissait lui-même, en 1984, la relative indigence de la recherche sur le sujet ${ }^{5}$. Ce constat, encore vrai aujourd'hui, est à l'origine de l'étude dont est tiré le présent article ${ }^{6}$. La question du local municipal est évoquée dans les ouvrages traitant des mai-

1. Agulhon, Maurice, "La Mairie, Liberté, Égalité, Fraternité ", NorA, Pierre (dir.), Les Lieux de mémoire, t. 1, La République, Paris, Gallimard, 1984, p. 168.

2. Article 136, § 1; MoRGAND, Léon, Commentaire de la loi du 5 avril 1884 surl'organisation et les attributions des conseils municipaux, Paris, Librairie administrative, 1902, t. 2, p. 281.

3. George, Jocelyne, Histoire des maires 1789-1939, Paris, Plon, 1989, p. 91.

4. SAFTA, Soraya et GAREVSKI, Tomislav, Ces belles mairies de France, Paris, Patrimoine Plus, 1995, p. 20.

5. AgulHon, Maurice, « La Mairie, Liberté, Égalité, Fraternité... ", art. cit., p. 190.

6. Cet article est la version synthétique d'un mémoire de maîtrise soutenu en septembre 2001 : GEFFroy, Denis, La Construction des Mairies dans l'arrondissement de Fougères 
res ou de l'administration locale, mais elle ne fait souvent l'objet que de quelques lignes. Par ailleurs, les mairies et hôtels de ville, dont les premières réalisations remontent au Moyen Âge, ont attiré le regard des historiens de l'art qui se sont en priorité intéressés aux plus beaux exemples du genre ${ }^{7}$.

Cette étude s'attache aux mairies plus modestes, celles des communes rurales, à l'échelle d'un arrondissement. En effet, l'arrondissement est un ensemble assez circonscrit pour qu'il soit possible de recueillir des informations précises sur les mairies de toutes les communes qui le composent, et assez vaste pour déceler d'éventuelles différenciations locales liées à l'appartenance politique ou à l'économie de chaque espace. Par ailleurs, le choix de cette entité territoriale garantit une certaine homogénéité dans l'application des directives étatiques et préfectorales par le sous-préfet. C'est dans cette optique qu'a été choisi l'arrondissement de Fougères, regroupant 57 communes au nord-est du département de l'Illeet-Vilaine, frontalier de la Normandie et du Maine ${ }^{8}$. Arrondissement rural et pauvre, dominé par une polyculture traditionnelle qu'Henri Goallou juge " retardataire et médiocre ${ }^{9}$ ". Campagne recouverte de landes, de forêts et d'un épais bocage ainsi décrite en 1846 par Amédée Bertin et Léon Maupillé :

"Aux seules maisons construites en pierres, qui ont remplacé les maisons en terre [du bassin rennais], on reconnaît que l'on est dans l'arrondissement de Fougères; les collines deviennent plus nombreuses, plus escarpées, les champs plus divisés, plus plantés, les clôtures qui entourent chaque champ sont plus élevées [...] toute la campagne devient un épais bocage ${ }^{10}$."

L'industrie est quasiment absente en dehors de Fougères ${ }^{11}$, l'exploitation du sous-sol granitique dans les cantons de Saint-Brice-en-Coglès et Louvigné-du-Désert conserve un caractère artisanal. Peu touchées par l'industrie, les communes de l'arrondissement trouvent en revanche dans l'amélioration des voies de communication et le commerce une source de développement économique. L'amélioration du réseau routier, sous le Second Empire notamment, et l'inauguration de la ligne de chemin de fer Vitré-Fougères en 1867, puis de celle reliant Antrain à Rennes, ouvrent ce pays. En outre, les marchés ruraux et les foires se multiplient. Les plus pro-

au XIX siècle, intérêt local et encadrement étatique, mémoire de maîtrise, Université de Rennes 2, dir. Patrick HARISMENDY, 2001, 257 p. dactyl.

7. Le titre de l'ouvrage de SAFTA, Soraya et GAREvski, Tomislav, Ces belles Mairies de France, en témoigne.

8. La sous-préfecture, Fougères, a été écartée de l'enquête puisqu'il s'agit d'une ville de 9100 habitants en 1851, centre de productions industrielles. L'étude porte donc sur 56 communes.

9. GoAllou, Henri, L'Évolution politique de l'Ille-et-Vilaine du 2 décembre 1851 au 5 janvier 1879, Thèse d'État, université de Rennes 2, 1971, p. 14.

10. Bertin, Amédée et MAUPILlE, Léon, Notice historique et statistique sur la Baronnie, la ville et l'arrondissement de Fougères, Rennes, Imprimerie de A. Marteville, 1846, p. 294.

11. Fougères abrite une industrie de confection de chaussures qui connaît son âge d'or dans la deuxième moitié du XIX ${ }^{\mathrm{e}}$ siècle. 
spères sont celles de Fougères, Antrain, Saint-Brice-en-Coglès, Louvigné-duDésert, Saint-Georges-de-Reintembault, Saint-Aubin-du-Cormier.

Sur le plan politique, le rayonnement politique de Fougères, cité républicaine, est nul, ce qui ne signifie pas que l'ensemble de l'arrondissement soit acquis aux conservateurs ${ }^{12}$. Une fracture politique qui s'exprime avec une certaine continuité depuis la Révolution partage l'arrondissement. D'une manière certes un peu schématique on distingue des régions de sensibilité " bleue " et d'autres de sensibilité "blanche ${ }^{13}$ ". Les deux cantons de Fougères - exception faite de la sous-préfecture - restent un fief conservateur, «c'est l'atmosphère du Maine qui continue de régner dans ce milieu royaliste et catholique [...], l'esprit de chouannerie continue de flotter dans ces campagnes ${ }^{14}$ ". En revanche, les cantons d'Antrain, de Saint-Aubin-duCormier et de Saint-Brice-en-Coglès votent républicain ${ }^{15}$. Le canton de Louvigné-du-Désert est plus partagé, sa frange occidentale est acquise aux républicains, sa frange orientale aux conservateurs. Cette dichotomie, qui souffre bien entendu de multiples exceptions, permet d'évaluer la pertinence du déterminant politique dans la construction d'une mairie ${ }^{16}$. Dernière observation, le clergé conserve dans l'ensemble de l'arrondissement une influence considérable, puisque selon Henri Goallou « en 1851, il n'existe en Ille-et-Vilaine aucune force capable de s'opposer à celle du clergé : ni les légitimistes, ni les libéraux, ni l'extrême gauche, ni l'enseignement ne peuvent rien ${ }^{17}$ ".

Dans ce contexte, le mot " mairie " s'avère de nature polymorphe. C'est le terme le plus fréquent dans les archives consultées et qui s'est imposé depuis la fin du XIX ${ }^{\mathrm{e}}$ siècle pour désigner le bâtiment où se tient l'administration municipale. Néanmoins l'expression " maison commune " est employée de manière équivalente quoique progressivement frappée d'une certaine désuétude. En effet, elle témoigne de la relative polyvalence, du caractère indéfini de l'édifice public, mais perd de sa pertinence à mesure que s'affirme la fonction de maire. L'expression " hôtel de ville " est très rare, elle a été relevée pour Antrain et Louvigné-du-Désert, mais elle induit une monumentalité étrangère aux édifices considérés. Mairies, maisons communes, ces deux formulations peuvent paraître abusives quand elles désignent une simple salle de réunion parfois accompagnée d'un cabinet pour les archives, le reste de l'édifice étant consacré au service scolaire.

12. Siegrfried, André, Tableau politique de la France de l'Ouest, Paris, Armand Colin, 1913, [rééd. Paris, Imprimerie nationale Éditions, 1995, p. 162].

13. Ces deux adjectifs ont été choisis à dessein pour leur relative imprécision, et pour souligner que l'on s'attache plus, à l'instar des travaux d'André Siegfried, à une sensibilité politique globale, qu'au vote circonstancié pour tel parti ou candidat

14. Siegrfried, André, Tableau politique..., op. cit., p. 161-162.

15. Blanchet, Jean-François, Approche de la vie électorale dans l'arrondissement de Fougères de 1874 à 1892, mémoire de maîtrise, université de Rennes 2, 1984, p. 97.

16. Cette hypothèse, énoncée par Maurice Agulhon, est à l'origine de notre étude. Néanmoins, à mesure que nos recherches avançaient, nous avons été amenés à reconsidérer son importance, au point d'en faire un objet d'interrogation secondaire.

17. Goallou, Henri, L'évolution politique de l'Ille-et-Vilaine..., op. cit., p. 89. 
À tel point qu'en fait de mairie-école, il serait plus juste de parler d' "écolemairie " pour rendre compte de la place occupée par la classe et le logement de l'instituteur ou de l'institutrice. En outre, il est fréquent que le dossier de construction de l'" école-mairie " ne fasse guère mention de la salle municipale. Cette absence explique en partie que les historiens n'aient pas perçu l'existence d'un local municipal. Dès lors, sans doute aurait-il été plus juste d'étudier ces bâtiments dans la pluralité de leurs affectations. Mais c'était accorder, une fois de plus, une place secondaire à la question du local municipal. Par ailleurs, si le modèle d'" école-mairie " est majoritaire, il n'est pas exclusif. Certaines communes associent la mairie et la justice de paix, ou la mairie et la halle, ou encore affectent à un édifice le seul service municipal. Par conséquent, le choix de privilégier le local municipal se justifie même si la construction des mairies reste liée à celle des écoles communales.

\section{Le " système politico-administratif local " et la construction des mairies}

La discrétion des historiens au sujet des mairies rurales s'explique entre autres par la relative pauvreté des sources touchant aux mairies, sources qui nécessitent souvent des dépouillements importants pour un résultat parfois décevant. Toutefois, les archives départementales d'Ille-et-Vilaine conservent des sources essentielles : la série $2 \mathrm{O}$ - qui concerne l'administration et la comptabilité communale - recueille en effet les dossiers de construction des mairies. En cas d'absence de celui-ci, le dossier de construction de la maison d'école révèle généralement l'existence d'une salle de mairie (qui n'est pas toujours mentionnée dans le titre du dossier). Ces dossiers, souvent incomplets, sont composés de la correspondance passive et active de la préfecture avec la municipalité (délibérations du conseil municipal notamment) et les différentes autorités concernées (le sous-préfet, le Conseil général, le Conseil local des Bâtiments civils, l'inspecteur primaire, l'inspecteur d'Académie, le ministère de l'Instruction publique, le receveur municipal), ainsi que des documents techniques (plans, devis) et officiels (affiches et procès-verbaux d'adjudications, procès-verbaux d'enquête de commodo et incommodo, actes de vente, décomptes définitifs des travaux). Cette correspondance, souvent volumineuse, offre des renseignements d'ordre factuel (l'allure du bâtiment, son prix, l'auteur des plans, les financements des travaux, etc.). Mais une lecture plus minutieuse permet de saisir l'attitude des différents acteurs, en particulier à travers les difficultés et les conflits qui surgissent.

L'ensemble de ces dossiers présente une lacune importante. Dans la plupart des cas leur examen ne permet pas de déterminer la situation de l'installation municipale avant la première acquisition ou construction d'un bâtiment affecté (en partie) au service de mairie. Les comptes de gestion (conservés dans la série 20), qui indiquent l'ensemble des recettes et dépenses annuelles de la commune, pallient ce manque. En effet, les comp- 
tes de gestion, qui se présentent à partir de la fin du Premier Empire sous la forme de cahiers pré-imprimés, accordent une ligne aux dépenses de " loyer et entretien de la maison commune ». Ils donnent en outre une idée des capacités financières de la commune, dont l'évolution a été mesurée pour quelques communes. Ainsi, cette source, qui a " pour l'instant fait l'objet de peu d'études ${ }^{18}$ ", s'avère très utile et permet de fructueux recoupements avec les informations tirées des dossiers de construction.

Ces sources écrites - manuscrites ou imprimées - sont complétées par des sources iconographiques. Aux plans de constructions figurant parfois dans les séries $2 \mathrm{O}$ ou E dépôt ADM, s'ajoutent quelques cartes postales anciennes répertoriées dans la série 6 Fi. Ces documents donnent une idée précise de l'architecture des bâtiments construits, et parfois de leur insertion dans le bourg.

L'essentiel des sources est d'origine administrative et émane des trois niveaux hiérarchiques différents (administration centrale, administration départementale, administration municipale), ce qui explique l'angle d'analyse choisi. D'une façon générale, la construction des mairies se situe à la rencontre de l'histoire de l'administration, de l'histoire politique telle qu'elle a pu être renouvelée par Maurice Agulhon, et de l'histoire de l'éducation. Mais cette recherche ne s'inscrit pas dans la perspective « agulhonienne " d'étude de la symbolique républicaine. D'une part nous ne disposons pas de travaux de recherche suffisamment précis sur la vie politique locale de l'arrondissement de Fougères. D'autre part, une telle problématique ne peut s'appliquer - si ce n'est a contrario - à des bâtiments dont la simplicité et l'antériorité de la construction contredisent l'idée même de mairie républicaine. Par conséquent, cette étude ressortirait plutôt à une histoire de l'administration locale et du patrimoine administratif, car il faut bien lier la municipalité à " son support matériel ${ }^{19}$ " .

À cela s'ajoute l'existence d'un " système politico-administratif local ", qu'avaient relevée Jean-Pierre Worms ${ }^{20}$ et Pierre Grémion ${ }^{21}$ dans leur contestation du schéma tocquevillien de l'administration française. L'administration devant composer avec les intérêts locaux et le pouvoir des notables, c'est dans ce système et cette rencontre que réside la réalité du pouvoir. Il convient donc d'analyser les relations qui se tissent entre les municipalités, la préfecture et les autres intervenants, à l'occasion de la construction d'une mairie. L'enjeu étant de savoir dans quelle mesure la construction ou l'acquisition d'un local municipal, appelons-le mairie, est déterminée par le processus d'émancipation municipale qui caractérise le XIX ${ }^{\mathrm{e}}$ siècle?

18. MAUREPAS, Arnaud de, Économie et finances au XIXe siècle. Guide du chercheur 17891870, Paris, Comité pour l'histoire économique et financière de la France, 1998, p. 384.

19. AgulHon, Maurice, «Imagerie civique et décor républicain dans la France du XIX ${ }^{\mathrm{e}}$ siècle ", Ethnologie française, 1975, 1, p. 40.

20. Worms, Jean-Pierre, "Le préfet et ses notables ", Sociologie du travail, 1966, 3, p. 249-275. 


\section{La précarité de l'installation municipale dans les premières décennies du $\mathrm{XIX}^{\mathrm{e}}$ siècle}

Les principales villes du royaume de France se dotent au Moyen Âge et à l'époque moderne d'institutions municipales et parallèlement d'hôtels de ville. De même, la Révolution, en transformant chaque paroisse rurale en commune, entité territoriale disposant d'un corps municipal chargé de l'administrer, crée en même temps un besoin d'édifices municipaux. Mais il faut attendre la Troisième République et la loi municipale du 5 avril 1884 pour que chaque commune soit tenue de disposer d'un local municipal, comme propriétaire ou comme locataire.

La nécessité d'un local municipal s'est fait sentir avant cette loi. Deux raisons sont avancées par les conseils municipaux et les autorités. Une mairie est à la fois un lieu de conservation des archives, du cadastre, des documents d'état civil, et un lieu de réunion du conseil municipal, dont les sessions se multiplient au long du siècle. Ce sont donc avant tout des besoins concrets qui poussent les communes à se doter de maison commune. Même si jusqu'aux lois de 1831 et 1837, l'administration communale, en particulier dans les petites communes est assez inerte, les municipalités de l'arrondissement de Fougères louent une " maison commune " dès les premières années du siècle. Cette « maison " se résume généralement à une pièce réservée au service municipal, dans une habitation particulière. Assez souvent, il semble que ce soit le maire qui accueille dans sa maison (gratuitement ou contre le versement d'un loyer) les activités de l'administration municipale. Jusqu'en 1812, date à laquelle apparaissent les cahiers de comptes pré-imprimés, le nom du propriétaire du local est indiqué. Quand il s'agit du maire, on trouve la mention "Payé au maire ", par conséquent une étude détaillée, portant sur la moitié de l'effectif (27 communes), a pu être menée. Sur les 53 noms de propriétaires recensés, 13 étaient maires (25\%). Ce chiffre brut, qui ne tient pas compte de la durée de location, peut être affiné en comptabilisant les années de location pour lesquelles le nom du propriétaire est connu. On obtient alors le chiffre de $31 \%$. Une telle installation présente d'incontestables avantages, mais il est possible que la demeure du maire (petit notable, paysan aisé) soit de toute façon une des seules du chef-lieu de la commune à être suffisamment grande pour accueillir les conseillers municipaux et les archives. En tout état de cause, l'installation de l'administration municipale reste précaire. Le local loué est soumis à des changements fréquents, préjudiciables à la bonne conservation des archives. L'étude précitée sur les premiers comptes de gestion révèle en effet de nombreux changements de propriétaires. Ainsi, la commune de Dompierre-du-Chemin verse en l'an XIII, un loyer de 20 francs à M. Quenier, l'année suivante un loyer de 32 francs au maire M. Lemonnier, en 1807 apparaît de nouveau le nom de M. Quenier, de 1808 à 1810 un loyer de 18 puis 15 francs est versé à M. Fleury, enfin, en 1811, le dernier propriétaire connu est de nouveau le maire, M. Lemonnier. Ces observations concernant la première décennie du XIX ${ }^{\mathrm{e}}$ siècle sont-elles susceptibles 
d'extrapolation? L'institution municipale n'en est alors qu'à ces balbutiements, l'instabilité est sans doute moins préjudiciable à cette administration récente et encore réduite à son minimum. Néanmoins, plusieurs indications suggèrent une certaine permanence. Ainsi, en 1855, le maire de Combourtillé avertit le préfet que " [la] mairie est très mal logée et sujette à des changements très souvent car quand on afferme, ce n'est pas pour plus de trois ans ". La construction ou l'acquisition d'un bâtiment propre est donc une solution plus convenable à une institution qui commence à s'affirmer.

\section{Un mouvement de constructions antérieur à la Troisième République}

Prescriptions étatiques et préfectorales poussent en ce sens. Leur influence peut être mesurée à partir d'une étude attentive de la chronologie du mouvement des constructions et acquisitions de mairies dans l'arrondissement de Fougères.

Une première chronologie, établie selon les régimes politiques, révèle l'ancienneté du mouvement. Il débute sous la Monarchie de Juillet, période au cours de laquelle 37 \% des communes deviennent propriétaires de leur local de mairie. Il se poursuit sous la Seconde République (9\%) et le Second Empire (32\%), si bien qu'à la veille de l'avènement de la Troisième République, 80 \% des communes possèdent déjà leur local municipal. En 1909, 96 \% des communes sont propriétaires, même s'il faut attendre 1927 pour que le taux de 100 \% soit atteint. Dans cette portion de département, la Troisième République n'a donc pas généralisé mais achevé un mouvement commencé sous la Monarchie de Juillet et qui se poursuit de façon soutenue sous les deux régimes suivants. Un tel constat remet en cause l'idée selon laquelle la Troisième République aurait donné une impulsion déterminante à la construction des mairies de communes rurales, par opposition à un Second Empire bâtisseur d'églises et de croix de mission. Mais cette chronologie fondée sur les césures classiques de l'histoire politique ne rend compte du mouvement que de manière superficielle. Et c'est sans doute surestimer l'impact des changements de régime sur un phénomène ayant des rythmes propres, qui transcendent en partie les régimes politiques du siècle.

À cet égard, les lois scolaires fournissent des repères plus pertinents, tant est fréquente l'association de la mairie et de l'école. Quatre dates rythment cette chronologie : 1833, 1858, 1867, 1883-1884. Le 28 juin 1833, la loi Guizot exige de toutes les communes de plus de 500 habitants qu'elles se dotent ou entretiennent une maison d'école. Beaucoup de communes, dans la nécessité de construire (ou d'acquérir) une école publique saisissent cette occasion pour ménager dans le nouvel édifice un espace destiné au service municipal. La loi municipale du 18 juillet 1837 quant à elle se contente d'inscrire au nombre des dépenses obligatoires «l'entretien, s'il y a lieu, de l'hôtel de ville ou du local affecté à la mairie " (art. 30) ${ }^{22}$. La circulaire du

21. Gremion, Pierre, Le Pouvoir périphérique, Paris, Seuil, 1976, 477 p. 
ministère de l'Instruction publique du 30 juillet 1858, en rappelant les normes relatives aux constructions scolaires (emplacement, dimensions de la salle de classe, logement de l'instituteur obligatoirement composé de trois pièces), marque une seconde étape. En 1867, le ministre de l'Instruction publique, Victor Duruy, impose la loi sur le dédoublement des écoles mixtes dans les communes de plus de 500 habitants, disjoignant ainsi la maison d'école de garçons de celle des filles. Cette loi donne une troisième impulsion au mouvement de construction des écoles (de garçons et de filles) dont bénéficient les mairies, comme celle de Saint-Georges-de-Chesné. Enfin, les années 1883-1884 constituent l'ultime étape. La loi du 20 mars 1883 impose aux communes de posséder en propre la maison d'école municipale, celles qui louaient ce local doivent acquérir ou construire une maison d'école. L'impulsion donnée par ce texte précède donc celle de la loi municipale du 5 avril 1884. Dans l'arrondissement de Fougères, la loi Guizot inaugure le mouvement de constructions/acquisitions des mairies (-écoles) puisque 59 \% des communes se dotent d'une mairie au cours de la période 1833-1857. De 1858 à 1866, le phénomène concerne $14 \%$ des communes de même que de 1867 à 1882, si bien qu'en 1884, 91 \% de communes de l'arrondissement possèdent leur bâtiment municipal ${ }^{23}$. Les prescriptions de l'État central, dans le domaine scolaire bien plus que dans le domaine municipal, influencent donc le mouvement de constructions des mairies, mais il faut aussi évaluer l'action de l'homme chargé de les faire appliquer : le préfet.

Un préfet se détache nettement par l'attention particulière qu'il a portée à ce problème, Paul Féart, en poste de juin 1858 à septembre 1864. Selon Typhaine Le Yoncourt, "Féart a en effet tenté d'instaurer en Ille-et-Vilaine une politique communale d'envergure ${ }^{24}$ ". Il témoigne à de nombreuses reprises, en 1858 et 1859, de l'attention qu'il porte à la question de l'installation des autorités municipales. Selon lui, " la question des bâtiments est l'une des plus importantes de l'administration ${ }^{25}$ ". Quelques mois après son entrée en fonction, constatant que "l'installation des municipalités [...] laisse beaucoup à désirer ${ }^{26}$ ", Féart lance une grande enquête sur le personnel et le matériel dont disposent les administrations municipales. Pour compléter les observations qu'il peut faire au cours de ses nombreuses excursions dans le département, il envoie un questionnaire à chaque maire. Les questions au sujet des bâtiments de mairies sont nombreuses et détaillées : « La commune possède-t-elle un hôtel-de-ville ou mairie? Quelle est l'importance de ce bâtiment? [...] La maison commune est-elle une propriété publique $^{27}$ ? ", etc. Cette circulaire est aussi l'occasion pour le pré-

22. Morgand, Léon, Commentaire de la loi du 5 auril 1884..., op. cit., p. 281.

23. Le dynamisme du mouvement de constructions au cours de la période 1858-1866 doit aussi beaucoup à l'action du préfet alors en charge du département. Cf. infra.

24. LE Yoncourt, Tiphaine, Le Préfet et ses notables en Ille-et-Vilaine au XIXe siècle, 18141914, Thèse droit, université Rennes I, dir. François BURDEAU, p. 243.

25. Arch. dép. d'Ille-et-Vilaine, $1 \mathrm{~N}$ 64, délibérations du Conseil général, session de 1859, rapports spéciaux du préfet, p. 102.

26. Ibidem, rapport du préfet devant le Conseil général, introduction, p. XxI. 
fet d'appeler les communes à se doter d'une mairie, en avançant que " toute autorité convenablement installée et publiquement soutenue peut facilement accomplir tous les devoirs de sa mission, [...] il est indispensable que l'installation de toutes les autorités municipales soit sérieusement organisée $^{28}$ ". Féart va même jusqu'à souhaiter : "Lorsque je visiterai désormais les populations rurales, je ne trouverai plus de communes sans mairie ${ }^{29}$." Un tel volontarisme, accompagné par une augmentation des subventions départementales, semble avoir été suivi d'effet, puisque huit communes prennent l'initiative de se doter d'un bâtiment municipal entre 1858 et 1860. La politique du préfet Féart a donc incontestablement accéléré le mouvement et explique en partie pourquoi le Second Empire a été, dans l'arrondissement de Fougères, une période riche en constructions/acquisitions de mairies.

Au-delà de cette chronologie générale, certaines diachronies locales, liées aux caractéristiques propres de chaque commune, apparaissent. À cet égard, les sympathies que la commune peut exprimer à l'égard des républicains ou de leurs adversaires légitimistes ne semblent pas être un facteur si déterminant, puisqu'en moyenne les communes de sensibilités "bleues " sont rentrées en possession de leur première mairie en 1856, tandis que les communes " blanches " attendaient 1862. En outre, à trop surestimer le facteur politique, on risque de ne pas percevoir l'influence de critères plus pragmatiques tels que la richesse de la commune, ou l'importance de sa population. D'une manière générale le mouvement de construction des mairies se diffuse des communes les plus riches vers les communes les plus pauvres, des plus populeuses vers les moins peuplées, des chefs-lieux de cantons vers les communes ordinaires. Mais, ces différents facteurs se renforcent ou s'annulent et des éléments contingents interviennent, en particulier le dynamisme du maire ou du curé. On trouve donc des communes "blanches " ou des communes pauvres qui acquièrent tôt dans le siècle leur mairie (Villamée, 1834; Saint-Ouen-le-Rouërie, 1843), des communes républicaines qui préfèrent louer leur local de mairie jusqu'à une date avancée (Vendel, 1921). Mais en dépit de ces disparités, la chronologie révèle la précocité du processus d'équipement.

\section{" Et le chemin est long du projet à la chose " (Molière)}

La construction ou l'acquisition d'un bâtiment de mairie est souvent l'aboutissement d'un processus long, difficile, parfois conflictuel, associant de nombreux intervenants (le maire, le conseil municipal, les plus imposés, la population, le curé et le député parfois, le receveur municipal, le souspréfet, le préfet, l'administration scolaire quand il s'agit d'une mairie-école, le Conseil général, le Conseil local des Bâtiments civils) et marqué par la tutelle préfectorale. Celle-ci s'exerce par des procédures d'autorisation et 
de contrôle : en amont, sur les plans (soumis au Conseil local des Bâtiments civils) et les modalités de financement du projet, ainsi que sur l'adjudication des travaux, et en aval au moment de la réception de l'édifice. Ce contrôle porte tant sur le fond que sur la forme et le respect de la procédure $^{30}$. L'administration municipale est ainsi dépendante des autorisations préfectorales. Pourtant « on se tromperait sur le caractère de la tutelle si l'on n'y voyait qu'un instrument du pouvoir, utilisé à des fins politiques. Elle fut aussi une indispensable école de formation des maires et c'est en grande partie grâce à elle que l'institution communale a pu se doter de bases solides et de pratiques administratives éprouvées ${ }^{31}$ ". L'administration préfectorale, tout en étant attentive au respect des procédures et des réglementations, encourage et soutient la construction ou l'acquisition d'un local municipal. Ainsi, en 1865, le préfet constatant que " la commune de SaintMarc-sur-Couesnon [...] ne possède pas encore de bâtiment scolaire [...], prie en conséquence M. le sous-préfet d'engager le maire à choisir un terrain convenable pour l'emplacement de la maison de mairie et d'école et de charger un architecte de la rédaction d'un projet de bâtiment à construire $^{32}$ ". Les sous-préfets quant à eux sont souvent amenés à prendre en charge la conduite de ces projets présentant par définition un caractère exceptionnel que tous les maires ne sont pas en mesure d'assumer.

Mais le pouvoir préfectoral ne peut faire abstraction des réalités locales. Ainsi les constructions ou acquisitions de mairies font apparaître " un système politico-administratif local " plus complexe. Les maires comme les conseils municipaux n'ont pas un comportement identique : "volontaristes " et " réfractaires " se répartissent assez équitablement. Ainsi, en 1870 le maire de Saint-Christophe-de-Valains, le comte de la Belinaye, assume lui même la plus grande partie des frais d'acquisition d'une mairie-école, alors que celui de Landéan, $M$. du Pontavice, adversaire du régime républicain et de sa politique de laïcisation, s'oppose dans la dernière décennie du siècle à la construction d'une nouvelle mairie-école. Mais la plupart du temps, les maires semblent se limiter au rôle de rouage administratif, contrairement au curé. Ainsi dans trois des six cas d'intervention cléricale, le desservant assume même la direction du projet (Bazouges-la-Pérouse, Fleurigné, Mellé), ce qui constitue une manifestation exemplaire du cléricalisme qui imprègne ces campagnes ${ }^{33}$. Mais le maire et le curé ne sont pas les seuls acteurs locaux. L'attitude des conseillers municipaux, des plus imposés et de la population est ambivalente. Ils expriment généralement le souhait de doter leur commune d'un bâtiment municipal mais sont avant tout guidés par le

29. Ibid., p. 212

30. Pour une description précise de la procédure : GEFFroy, Denis, La Construction des Mairies dans l'arrondissement de Fougères..., op. cit., p. 40-45.

31. ChANDERnAGOR, André, Les Maires en France, XIX'-XXe siècle. Histoire et sociologie d'une fonction, Paris, Fayard, 1993, p. 125.

32. Arch. dép. d'Ille-et-Vilaine, 2 O/écoles/Saint-Marc-sur-Couesnon, lettre du préfet au sous-préfet, le 26 août 1865. 
souci de limiter au maximum la dépense qui reviendra à leur charge. Cela donne parfois lieu à d'interminables marchandages qui retardent la réalisation du projet. En effet, la longueur de l'instruction des projets est un trait caractéristique, ainsi le projet de construction d'une maire-école à Romagné date de 1854, mais le service de mairie ne pourra s'y installer qu'en 1877. De même la commune du Tiercent attend 21 ans avant de devenir propriétaire de sa mairie-école (1850-1871). Le temps moyen, pour la construction d'un bâtiment neuf, entre le début de l'instruction du projet et la réception du bâtiment est d'environ sept ans ${ }^{34}$. Plusieurs facteurs expliquent ce délai : la lourdeur de la procédure soumise à de nombreux contrôles de la préfecture, du Conseil local des Bâtiments civils et parfois de l'administration scolaire, les dossiers lacunaires qu'il faut renvoyer à la commune pour être complétés, les difficultés de financement.

En effet si la tutelle administrative limite la liberté des communes en matière de construction municipale, leurs capacités financières constituent une contrainte substantielle. À cet égard, l'amélioration de l'installation municipale doit beaucoup à son association avec l'école. Non seulement elle bénéficie indirectement de l'aide de l'État pour les constructions scolaires - il ne subventionne que la partie du bâtiment destinée au service scolaire, évaluée généralement à 90 \% de la dépense - mais le souhait d'améliorer les conditions matérielles de l'enseignement primaire a souvent fourni l'opportunité aux communes de devenir propriétaires de leur local municipal. En effet, plus que l'installation matérielle de l'administration municipale, c'est l'amélioration du service scolaire qui est souvent à l'origine du projet. Sur les 39 communes pour lesquelles on peut connaître les motivations de la construction, 32 font référence au service scolaire : soit elles ne possèdent pas de maison d'école, soit le propriétaire de la maison louée souhaite en retrouver la jouissance, soit le bâtiment occupé est exigu, insalubre, ou vétuste. Mais cette association se traduit par un contrôle étatique accru, exercé par l'inspecteur primaire et l'inspecteur d'Académie, qui veillent au respect des normes strictes qui réglementent les constructions scolaires. C'est donc avant tout par souci d'économie et par commodité - l'instituteur faisant souvent office de secrétaire de mairie - que les communes associent la mairie et l'école dans un même bâtiment, et ce depuis la loi Guizot. Le pragmatisme, avant l'idéologie, explique cette association. Ainsi, afin d'obtenir une subvention du Ministère de l'Instruction publique, la commune d'Antrain décide que la maison acquise en 1834 pour abriter les services de mairie et de justice de paix " servirait aussi d'école mutuelle pour cent élèves et au logement de l'instituteur ${ }^{35}$ ". Mais la dépense n'en reste pas moins considérable, deux à trois fois le budget annuel selon les communes. Le coût moyen des constructions neuves

33. Dans les trois autres cas (Monthault, Poiley, Le Tiercent) le curé se contente d'exprimer au préfet son soutient au projet présenté par la commune.

34. Ce chiffre indicatif concerne les projets de construction pour lesquels, les dates des premières étapes de l'instruction et de la réception sont connues. 
s'élève à 12500 francs, contre 6800 francs pour l'acquisition et l'appropriation d'un édifice existant, avec une tendance à la hausse au cours du siècle. Or, les difficultés de financement sont réelles, les budgets communaux faibles et très sollicités par la construction des écoles, chemins vicinaux, presbytères et églises. Par exemple :

" La commune de Marcillé a eu à supporter depuis quelques années des sacrifices assez lourds, qui ont complètement épuisé ses ressources : elle a d'abord fait construire une nouvelle église à ses propres frais et à, l'aide de souscriptions, fait ouvrir trois chemins vicinaux dont deux ne sont pas encore achevés, pour lesquels elle s'est imposée de 10 centimes pendant 30 ans, et ensuite le mur d'enceinte du cimetière refait à neuf, et divers travaux de nivellement qui ne coûteront pas moins de 2000 francs, de sorte que la commune se trouve aujourd'hui dans une grande pauvreté ${ }^{36}$."

Les efforts consentis, non sans résistance, par les communes sont donc importants. Les ressources fiscales ordinaires ne suffisent pas, la commune doit vendre tout ou partie de ses terrains communaux, procéder à des souscriptions, et parfois se résoudre à s'imposer extraordinairement. Certains élus importants, le maire, le député, le conseiller général font parfois un don. Même si la commune assume l'essentiel de la dépense, elle reste fortement dépendante de l'administration préfectorale qui dispose par l'octroi des subventions départementales d'un pouvoir de pression non négligeable. Faiblesses des ressources, contrôles multiples, les modalités de financement sont extrêmement contraignantes. Elles influencent par conséquent l'architecture des édifices réalisés.

\section{Simplicité et homogénéité de l'architecture : entre idéologie et pragmatisme}

Les mairies de l'arrondissement de Fougères se caractérisent avant tout par leur extrême modestie, en dépit de l'association quasi-systématique du service municipal avec un autre service. Sur les 68 mairies étudiées, 56 sont des mairies-écoles (82\%), principalement des mairies-écoles de garçons $^{37}$. À partir de 1867, tous les projets présentés dans l'arrondissement sont des mairies-écoles de garçons. En effet, l'association du service de mairie avec l'école des filles, bien que n'étant pas formellement interdite, apparaît souvent problématique, pour des raisons qui tiennent à l'exclusion des femmes de la sphère politique et au recours privilégié à des institutrices congréganistes pour l'instruction des filles. L'affirmation du caractère laïc du pouvoir municipal serait incommodée par un tel voisinage. La salle de classe et le logement de l'instituteur (ou de l'institutrice) constituent la majeure part de l'édifice, mais le service scolaire souffre parfois de la proximité de la mairie et du va-et-vient qu'elle suscite. Afin de

35. Délibération du conseil municipal citée par JARRY, Alphonse, Histoire moderne de la ville et paroisse d'Antrain, Fougères, Imprimerie Pierre Saffray, 1936, p. 139.

36. Arch. dép. d'Ille-et-Vilaine, 2 O 165/16, délibération du conseil municipal, le 29 juin 1890 . 
remédier à cette nuisance s'impose, sous le Second Empire, le modèle " en $\mathrm{T}$ " pour reprendre l'expression utilisée dans les dossiers de constructions $^{38}$. Un bâtiment principal sur deux niveaux est destiné au logement de l'instituteur et à la mairie, la salle de classe est accolée d'équerre à la façade intérieure de celui-ci. Ce "plan en T " permet ainsi une nette séparation des deux services. Le même souci de distinction s'impose aux mairies-halles (Bazouges-la-Pérouse, Saint-Georges-de-Reintembault) et aux mairies-justices de paix (les quatre chefs-lieux de cantons, Antrain, Louvigné-duDésert, Saint-Aubin-du-Cormier, Saint-Brice-en-Coglès). En dépit des difficultés que peuvent poser la cohabitation de deux services parfois fort différents, les bâtiments exclusivement réservés à l'administration municipale sont très rares. On ne dénombre que 6 bâtiments de ce type (Bazouges-la-Pérouse, Saint-Aubin-du-Cormier, La Selle-en-Luitré, Vendel, et les deux mairies successives de Montours), construits pour la plupart dans les dernières décennies du siècle, mais pas nécessairement dans les plus communes les plus importantes.

Le Conseil local des Bâtiments civils ${ }^{39}$, organe chargé d'examiner les projets de travaux à faire pour le compte des institutions publiques du département (préfecture, conseil général, communes...) veille non seulement à la fonctionnalité des édifices, à la séparation des différents services qu'il abrite, mais porte également une attention particulière à l'architecture extérieure des mairies, la façade notamment. Ce conseil veille selon ses mots à « la convenance et à la dignité des édifices publics ${ }^{40}$ " et invoque fréquemment la notion de "bon goût ", de "bon effet ». Encadrée par cette institution normative, limitée par la faiblesse financière des communes, influencée par des plans-modèles qui circulent à l'échelle nationale dès le Second Empire, la liberté des architectes est restreinte. Mais leur formation, empreinte de références antiques, ne les portent guère à se détacher du style néoclassique - toutes proportions gardées - qui caractérise l'architecture communale à l'échelle nationale, alors que dans le domaine religieux prévaut le style néogothique dès les années 1845. Selon le Conseil local des Bâtiments civils, le bâtiment de mairie doit être simple, régulier, sobre, voire sévère, mais reconnaissable. Bref, inspirer le respect pour l'institution qu'il abrite. Ces contraintes contribuent à l'homogénéité qui caractérise les mairies-écoles de l'arrondissement, d'autant qu'un même architecte est souvent l'auteur de plusieurs édifices. Les architectes fougerais Jourdin et Prioul ont respectivement à leur actif 8 et 9 constructions de

37. Ce chiffre regroupe l'ensemble des mairies construites ou acquises au cours du XIX siècle par les communes de l'arrondissement de Fougères.

38. Sur les 34 mairies-écoles dont on connaît l'architecture extérieure, 21 (62 \%) ont un " plan en T ". Si on constate la diffusion de ce modèle dans l'arrondissement de Fougères à partie du Second Empire, nous n'avons pas pu en déterminer l'origine exacte. Il s'agit vraisemblablement d'un plan-modèle élaboré à l'échelle nationale.

39. Pour une étude détaillée du Conseil local des Bâtiments civils d'Ille-et-Vilaine, se reporter à VEILLARD, Jean-Yves, Rennes au XIX siècle, architecture, urbanisme et architectes, Rennes, Éditions du Thabor, 1978, 520 p. 
mairies dans l'arrondissement. Ainsi les mairies de l'arrondissement de Fougères prennent généralement la forme d'un bâtiment de base rectangulaire s'élevant sur deux niveaux, qui s'articule autour d'un axe central composé de l'entrée principale et de la fenêtre centrale du premier étage et d'un fronton, d'une lucarne ou d'un modeste campanile. La banalité de ces constructions est tout juste relevée par des jeux de matériaux (pierres de granit taillé, briques encadrent les ouvertures tandis que des moellons en granit remplissent les surfaces). Les éléments décoratifs sont rares, aucune inscription d'inspiration républicaine n'a été relevée, à commencer par la devise nationale «Liberté, Égalité, Fraternité ». Seules les mairiesjustices de paix des chefs-lieux de cantons se singularisent quelque peu. De dimensions plus imposantes que les mairies-écoles de villages, elles cultivent une certaine monumentalité. Celle de Saint-Aubin-du-Cormier associant brique, granit et tuffeau, est composée d'un large corps central coiffé d'un fronton richement orné, et de deux ailes latérales, mais cet exemple constitue une exception.

La même simplicité se retrouve dans l'aménagement intérieur de la mairie. Le service municipal ne dispose le plus souvent que d'une ou deux pièces pour accueillir les réunions du conseil municipal et pour conserver les archives, registres et autres documents nécessaires à l'administration de la commune. L'ameublement est souvent réduit au minimum : une table, quelques chaises ou bancs, une armoire, souvent de mauvaise qualité. L'installation est donc assez rudimentaire, ce qui n'empêche pas certaines communes de posséder un drapeau ou un buste de souverain. Une seule effigie de Marianne a été relevée, un don du député radical Le Hérissé à sa commune natale d'Antrain.

Antériorité du mouvement, modestie des réalisations, ces conclusions nuancent et complètent donc certaines des idées traditionnellement formulées à l'endroit des mairies de village. Néanmoins, elles n'ont pas toutes vocation à la généralisation. Ainsi, la relative exiguïté et pauvreté des communes de l'arrondissement de Fougères ne permettent pas aux communes de se doter de mairies imposantes. Le passé chouan de certaines d'entre elles, et la permanence de cette sensibilité dans le soutien au courant légitimiste, induit une tradition d'opposition à l'État central en général, et à la République en particulier, qui contribue certainement à la passivité ou à l'opposition de certaines communes. En outre, en ces terres de cléricalisme, une mairie triomphante qui se poserait en rivale de l'église serait pour le moins incongrue. En revanche, la location de chambres municipales depuis le début du XIX ${ }^{\mathrm{e}}$ siècle et l'ancienneté du mouvement de construction observé dans l'arrondissement de Fougères, sont deux observations susceptibles d'être étendues à l'ensemble du territoire national.

Cette étude invite donc à reconsidérer l'identification de ces bâtiments au régime républicain. En assignant à l'école le rôle de républicaniser les campagnes, et en faisant de la mairie le symbole de cette réussite, les 
mairies-écoles sont devenues un attribut de la Troisième République. Mais, tel ne fut pas toujours le cas. La construction d'un local propre à l'administration municipale, avant l'obligation législative de 1884, est le signe d'une certaine émancipation du pouvoir municipal à l'égard des notables traditionnels et traduit une prise de conscience par les habitants de leur existence en tant que communauté politique. Pourtant, compte tenu de l'implication de l'État à toutes les étapes du projet, on ne peut faire de la mairie le seul symbole d'une liberté communale, qui du reste n'existe pas en France. La mairie est aussi un signe de la présence du régime, et au-delà, de l'État au village. Ainsi, les mairies participent au mouvement de descente de l'État vers les masses rurales, et d'intégration de la commune à l'espace national. Par conséquent, si la mairie est en quelque sorte une personnification de la commune et de son émancipation, elle l'incarne en tant que cellule administrative et cellule politique intégrée et dépendante d'un espace plus vaste, celui de la Nation.

\section{Mairie-Justice de paix de Louvigné-du-Désert, photographie, date inconnue}

(Arch. dép. d'Ille-et-Vilaine, 6 Fi/35/Louvigné-du-Désert/3)

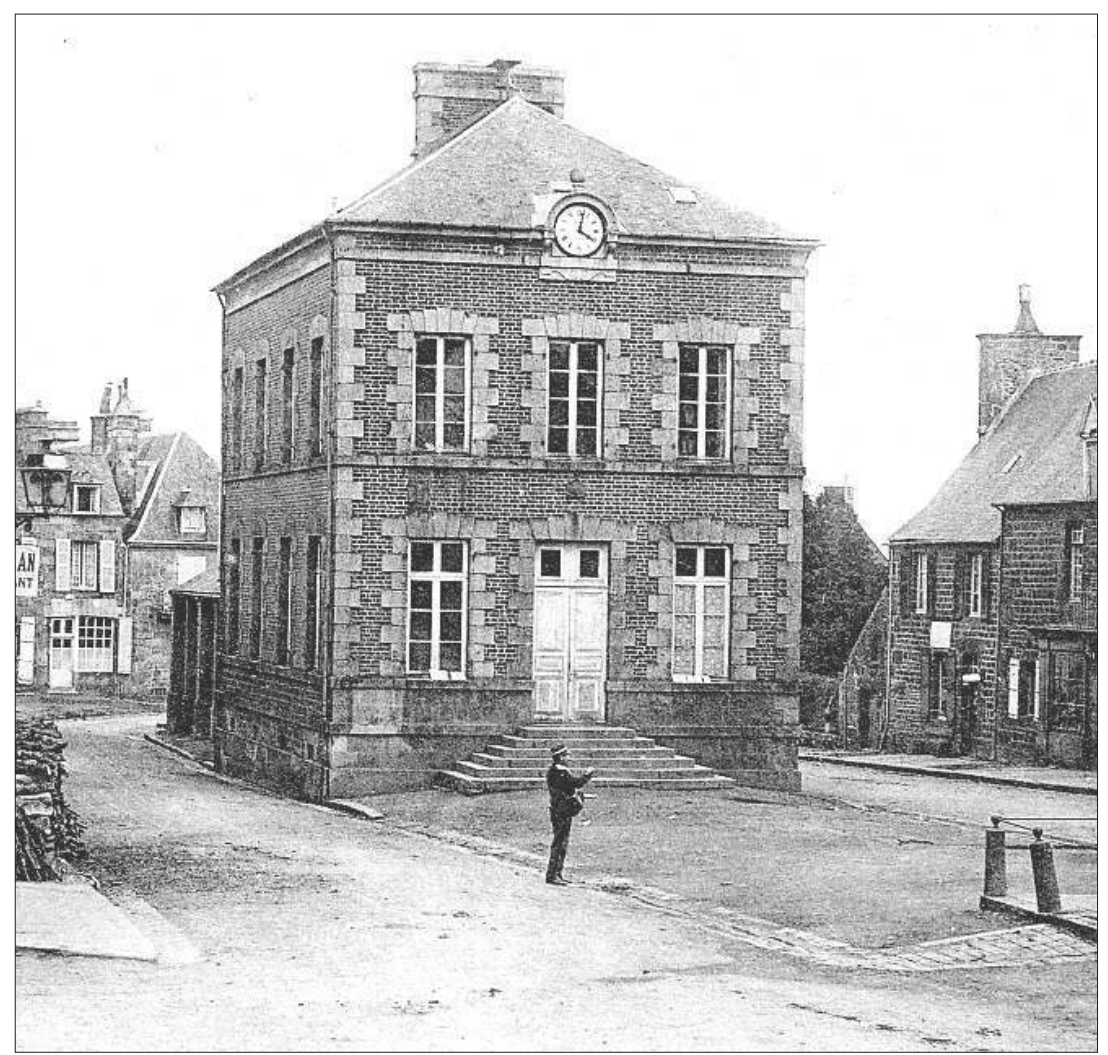


Mairie-École de Romagné, photographie, date inconnue

(Arch. dép. d'Ille-et-Vilaine, 6 Fi/35/Romagné/3)

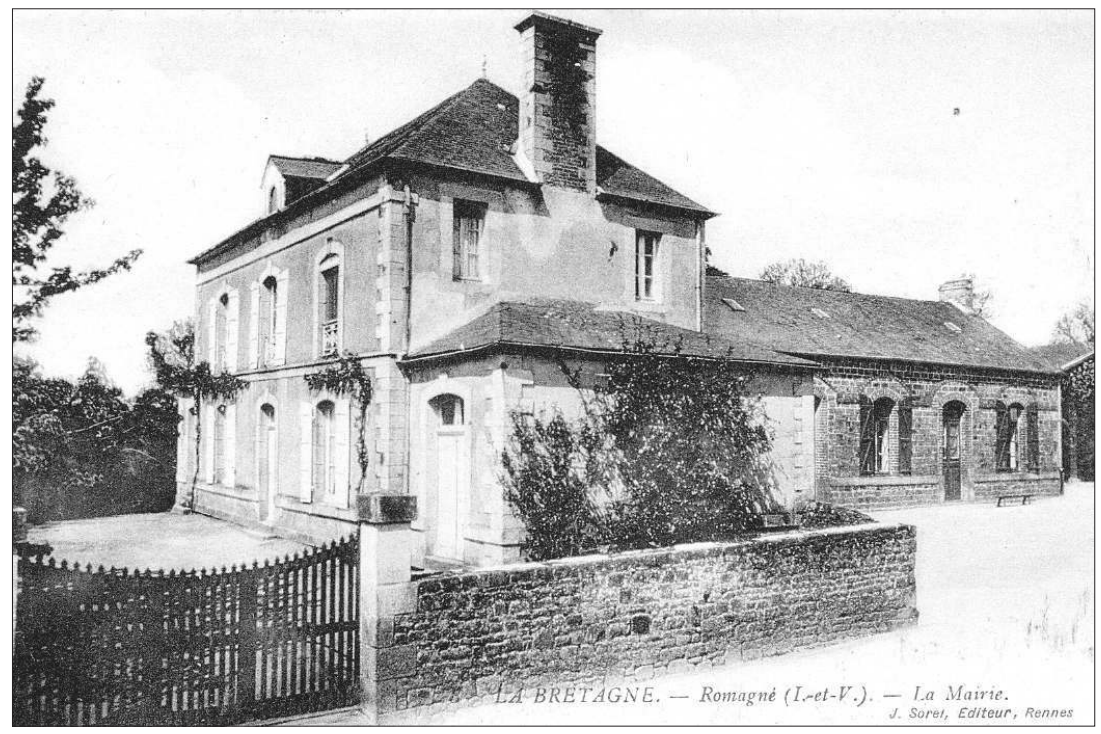

\section{Mairie-école de Saint-Georges-de-Chesné, plan en élévation, 1876, architecte Prioul}

(Arch. dép. d'Ille-et-Vilaine, E dépôt ADM/Saint-Georges-de-Chesné/13)

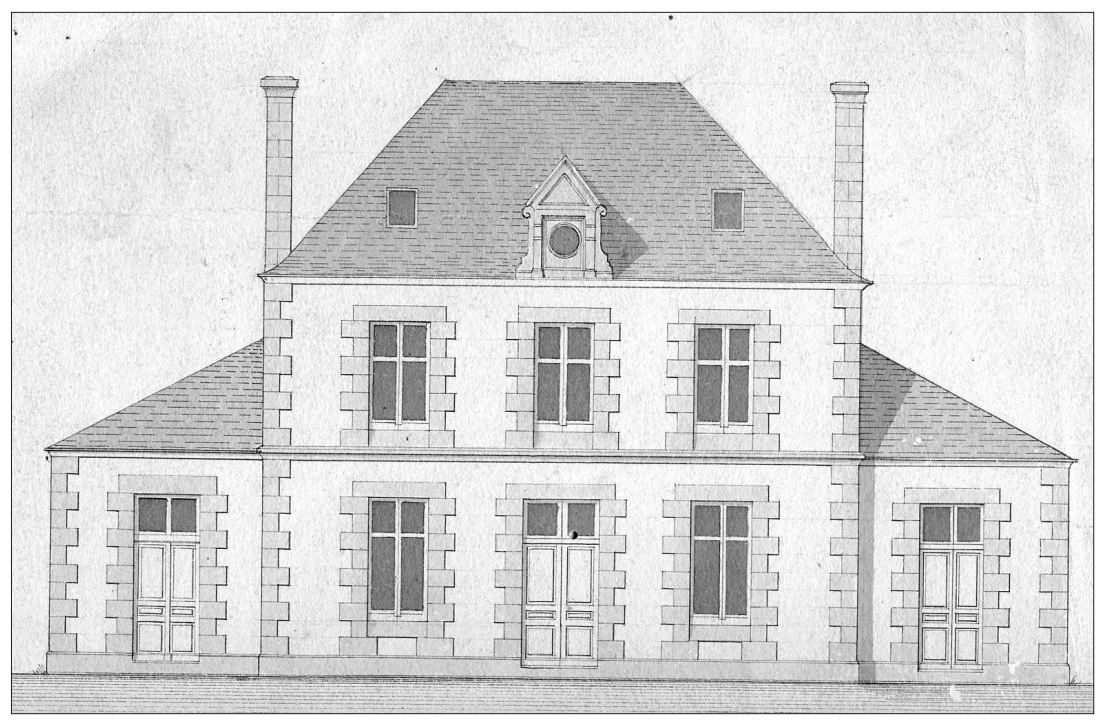

40. Arch. dép. d'Ille-et-Vilaine, 4 N 6, recueil délibération du Conseil local des Bâtiments civils, séance du 8 août 1866 . 
La Mairie avant la République

Les communes de l'arondissement de Fougères au XIX siècle

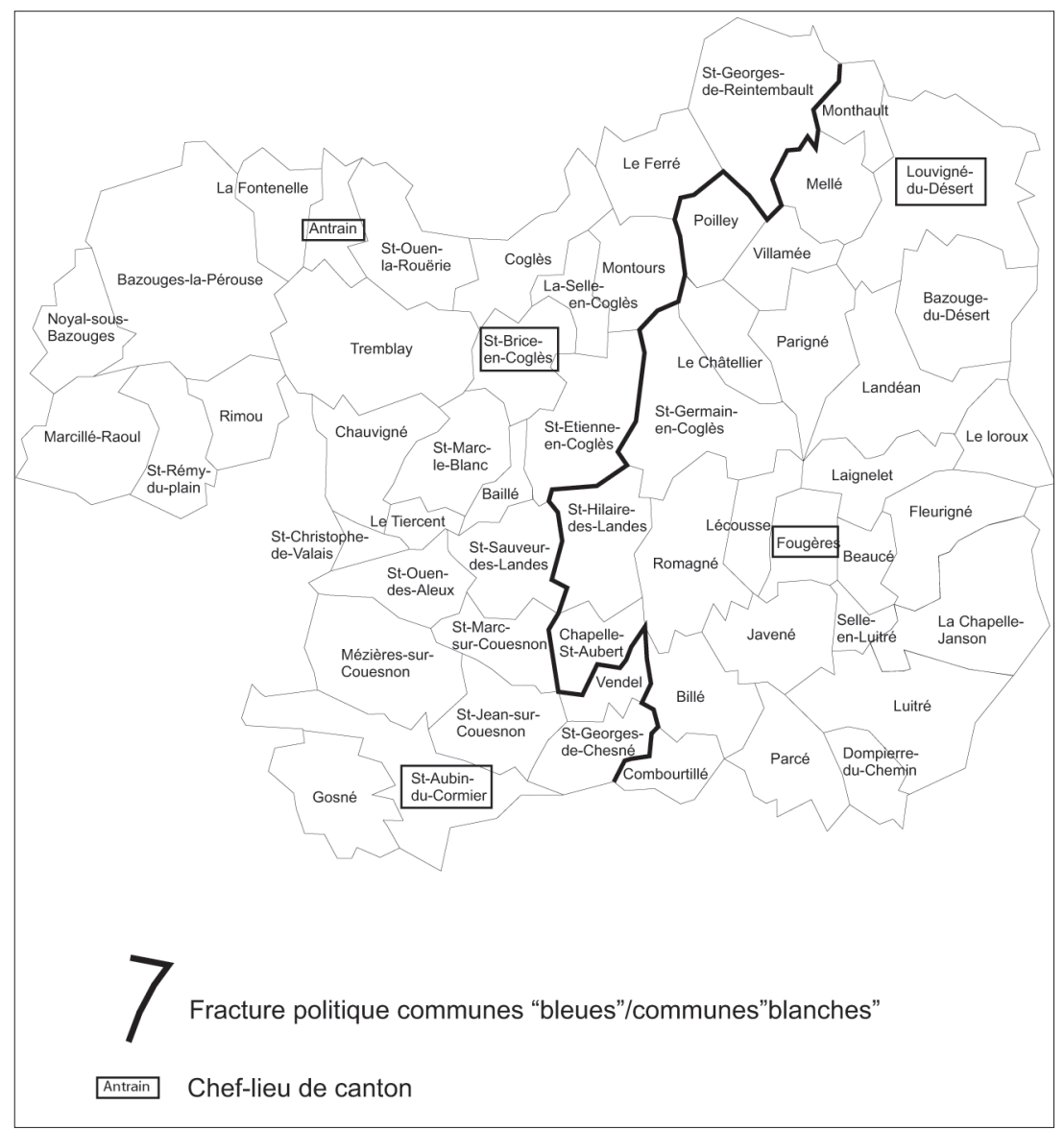


Chronologie et mode d'appropriation de la première génération de mairies de l'arondissement de Fougères

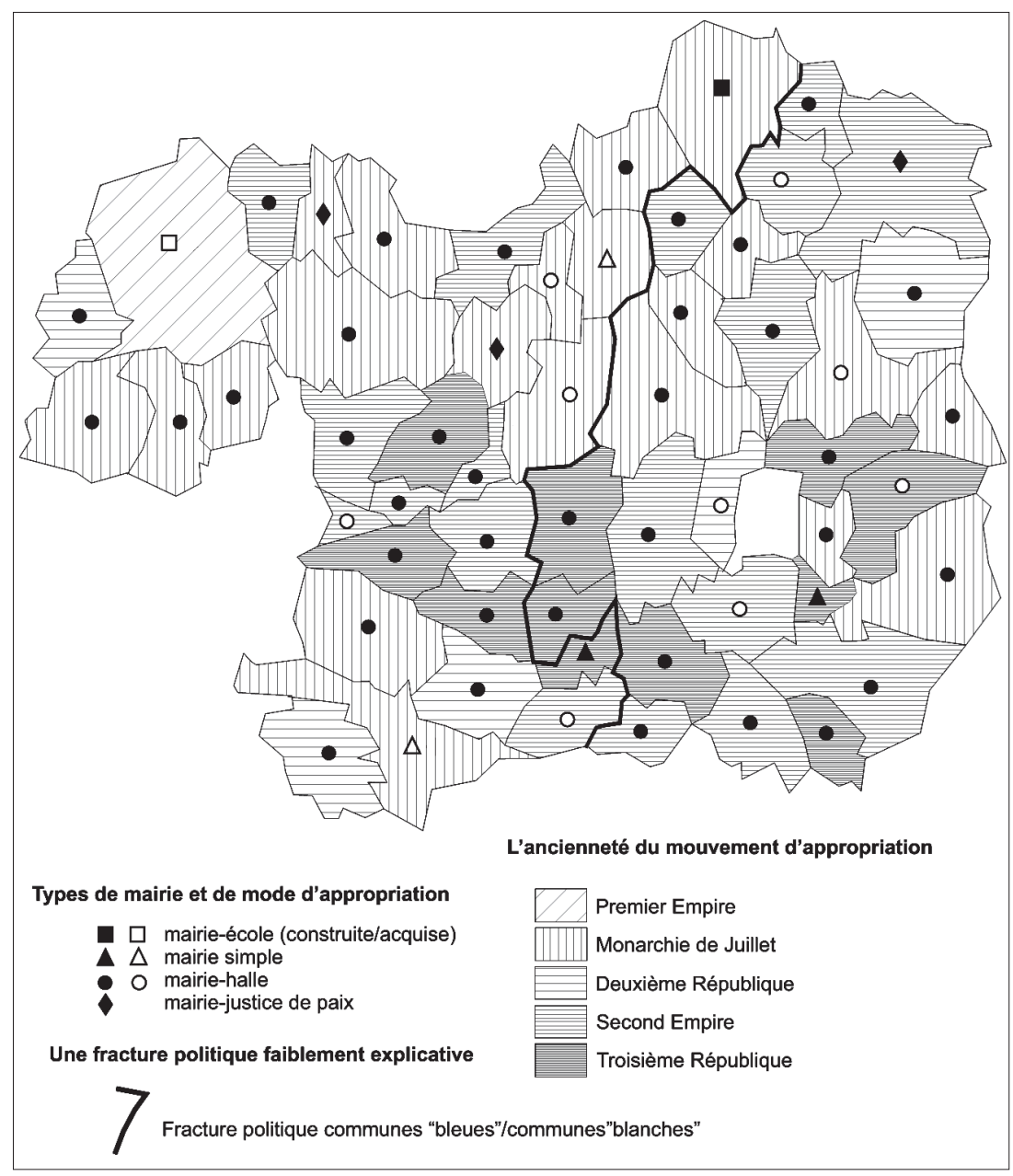




\section{RESUME}

Entamé sous la monarchie de Juillet, le mouvement de construction des mairies est presque achevé à la veille de la III ${ }^{\mathrm{e}}$ République. Avant de devenir un symbole républicain, la mairie apparaît avant tout comme un outil au service de l'amélioration d'une administration municipale encore rudimentaire. D'où les efforts constants des représentants de l'État central pour imposer à des communes souvent réticentes, la construction ou l'acquisition d'une maison, abritant la mairie et l'école.

\section{ABSTRACT}

During the July Monarchy, the movement of town halls' building began and yet was almost ended on the eve of the Third Republic. Before becoming a republican symbol, town halls appeared as a means to improve a still rudimentary local administration. That explains the steadfast effort of state officers to impose on reluctant villages the building or the purchase of a house to install the town council and the school. 
